\title{
THE EXTREME SCATTERING EVENT TOWARD PKS 1741-038: VLBI IMAGES
}

\author{
T. JOSEPH W. LAZIO \\ Naval Research Laboratory, Code 7213, Washington, DC 20375-5351; lazio@rsd.nrl.navy.mil
}

A. L. FEY

United States Naval Observatory, Code EO, 3450 Massachusetts Avenue NW, Washington, DC 20392-5420; afey@alf.usno.navy.mil

BRIAN DENNISON

Physics Department, Robeson Hall, Virginia Polytechnic Institute, Blacksburg, VA 24061-0435; dennison@astro.phys.vt.edu

F. MANTOVANI

Istituto di Radioastronomia del CNR, Via Gobetti 101, I-40129, Bologna, Italy; fmantova@rossem.ira.bo.cnr.it

J. H. SimONETTI

Physics Department, Robeson Hall, Virginia Polytechnic Institute, Blacksburg, VA 24061-0435; jhs@astro.phys.vt.edu

ANTONIO ALBERDI

Instituto de Astrofísica de Andalucía, CSIC, Apdo. 3004, Granada, Spain; alberdi@laeff.esa.es

A. R. FOLEY

Netherlands Foundation for Research in Astronomy, Postbus 2, NL-7990 AA, Dwingeloo, The Netherlands; tony.foley@nfra.nl

R. FIEDLER

Naval Research Laboratory, Code 7261, Washington, DC 20375-5351; Fiedler@sealab.nrl.navy.mil

M. A. GARRETT

Joint Institute for VLBI in Europe, Postbus 2, NL-7990 AA, Dwingeloo, The Netherlands; mag@jive.nfra.nl

HISASHI HIRABAYASHI

Institute of Space and Astronautical Science, 3-1-1 Yoshinodai, Sagamihara, Kanagawa 229, Japan; hirax@vsop.isas.ac.jp

D. L. JAUNCEY

Australia Telescope National Facility, Epping, New South Wales 2121, Australia; djauncey@atnf.csiro.au

K. J. JoHNSTON

United States Naval Observatory, 3450 Massachusetts Avenue NW, Washington, DC 20392; kjj@astro.usno.navy.mil

JON MARCAIDE

Departamento de Astronomía, Universitat de Valencia, E-46100 Burjassot, Valencia, Spain;jmm@vlbi.daa.uv.es

Victor Migenes

Departamento de Astronomia, Universidad de Guanajuato, Apartado Postal 144, 36000 Guanajuato, Gto, México; vmigenes@cuevano.astro.ugto.mx

G. D. NiCOLSON

Hartebeesthoek Radio Astronomy Observatory, P.O. Box 443, 1740 Krugersdorp, South Africa; george@bootes.hartrao.ac.za

AND

TIZIANA VENTURI

Instituto di Radioastronomia CNR, via Gobetti, 101, 40129 Bologna, Italy; tventuri@ira.bo.cnr.it

Received 1999 July 13 ; accepted 1999 October 19

\begin{abstract}
We report multiepoch VLBI observations of the source PKS 1741-038 (OT 068) as it underwent an extreme scattering event (ESE). Observations at four epochs were obtained, and images were produced at three of these. One of these three epochs was when the source was near the minimum flux density of the ESE, the other two were as the flux density of the source was returning to its nominal value. The fourth epoch was at the maximum flux density during the egress from the ESE, but the VLBI observations had too few stations to produce an image. During the event the source consisted of a dominant, compact component, essentially identical to the structure seen outside the event. However, the source's diameter increased slightly at $13 \mathrm{~cm}$, from near 0.6 mas outside the ESE to near 1 mas during the ESE. An increase in the source's diameter is inconsistent with a simple refractive model in which a smooth refractive lens drifted across the line of sight to PKS 1741-038. We also see no evidence for ESEinduced substructure within the source or the formation of multiple images, as would occur in a strongly refractive lens. However, a model in which the decrease in flux density during the ESE occurs solely because of stochastic broadening within the lens requires a larger broadening diameter during the event than is observed. Thus, the ESE toward PKS 1741-038 involved both stochastic broadening and refractive defocusing within the lens. If the structure responsible for the ESE has a size of order $1 \mathrm{AU}$, the level of scattering within an ESE lens may be a factor of $10^{7}$ larger than that in the ambient medium. A filamentary structure could reduce the difference between the strength of scattering in the lens and ambient medium, but there is no evidence for a refractively induced elongation of the source. We conclude that, if ESEs arise from filamentary structures, they occur when the filamentary structures are seen lengthwise. We are able to predict the amount of pulse broadening that would result from a
\end{abstract}


comparable lens passing in front of a pulsar. The pulse broadening would be no more than $1.1 \mu \mathrm{s}$, consistent with the lack of pulse broadening detected during ESEs toward the pulsars PSR B1937+21 and PSR J1643-1224. The line of sight toward PKS 1741-038 is consistent with a turbulent origin for the structures responsible for ESEs. The source PKS 1741-038 lies near the radio Loop I and is seen through a local minimum in $100 \mu \mathrm{m}$ emission.

Subject headings: ISM: general - quasars: individual (1741-038) — radio continuum: ISM scattering

\section{INTRODUCTION}

Extreme scattering events (ESEs) are a class of dramatic changes in the flux density of radio sources (Fiedler et al. $1994 a)$. They are typically marked by a decrease $(\gtrsim 50 \%)$ in the flux density near $1 \mathrm{GHz}$ for a period of several weeks to months, bracketed by substantial increases, viz., Figure 1 . Because of the simultaneity of the events at different wavelengths, the timescales of the events, and light-travel time arguments, ESEs are likely due to strong scattering by the Galactic interstellar medium (ISM; Fiedler et al. 1987a; Romani, Blandford, \& Cordes 1987). First identified in the light curves of extragalactic sources, ESEs have since been observed during a timing program of the pulsars PSR B1937+21 (Cognard et al. 1993; Lestrade, Rickett, \& Cognard 1998) and PSR J1643 - 1224 (Maitia, Lestrade, \& Cognard 1998).

ESEs are generally ascribed to be the result of strong interstellar refraction by discrete ionized structures (Fiedler et al. 1987a; Romani et al. 1987; Clegg, Fey, \& Lazio 1998), though Fiedler et al. (1994a) developed a model in which ESEs are due to stochastic broadening of source diameters. The extent to which refractive defocusing or stochastic broadening dominates during an ESE may be determined through VLBI imaging of a source undergoing an ESE. If refractive defocusing dominates, the position of the source may wander, and the shape of the lens or substructure within the lens may produce distortions in the appearance of the source; if the refraction is strong enough, the source may be multiply imaged (Cordes, Pidwerbetsky, \& Lovelace 1986; Clegg et al. 1998). If stochastic broadening dominates, the source structure should remain largely unchanged except for an increase in its diameter. The extent to which one or the other of these mechanisms dominates may also provide clues to the origin of these lenses. To date, the only observational constraints on the ESE mechanism-besides the light curves - have been the lack of pulse broadening (as might be expected from a stochastic broadening model) and a variation in the pulse time of arrival (as would be expected from a refractive defocusing model) during the ESEs toward PSR B1937 + 21 (Cognard et al. 1993; Lestrade et al. 1998) and PSR J1643-1224 (Maitia et al. 1998). Of course, it need not be the case that the ESE-like events observed toward these pulsars resulted from the same kind of structure responsible for the ESEs toward extragalactic sources.

This paper reports the first VLBI observations of a source (PKS 1741-038, OT 068) while it was undergoing an ESE. In $\S 2$ we describe the observations, in $\S 3$ we discuss the implications of our observations, and in $\S 4$ we present our conclusions and suggestions for future work.

\section{OBSERVATIONS}

Figure 1 shows a portion of the $2.25 \mathrm{GHz}$ light curve of PKS 1741-038 as obtained by the US Navy's extragalactic source monitoring program at the Green Bank Interferometer (GBI) (Fiedler et al. 1987b; Waltman et al. 1991; Waltman et al. 2000, in preparation). Clearly evident is an approximately $50 \%$ decrease in the source's flux density. The minimum occurred on or near 1992 May 25 (JD 2,448, 768.264), and the ESE is nearly symmetric about this epoch. We show only a portion of the light curve in order to focus on the ESE. The complete GBI light curve of PKS 1741-038, extending from 1983 to 1994, has been published previously (Clegg et al. 1998).

The epochs of observations are indicated in Figure 1, and the observing log is given in Table 1 . Also shown are the GBI flux-density measurements closest in time to the VLBI observations. While the GBI measurements were not simultaneous with the VLBI observations, the largest elapsed time between the GBI and VLBI observations was no more than 1 day. All observations were conducted in VLBI Mk II mode and recorded left circular polarization only. As part of this program, observations were also obtained on 1993 June 2, well after the ESE concluded.

Shen et al. (1997) conducted $6 \mathrm{~cm}$ VLBI observations in 1992 November. Fey, Clegg, \& Fiedler (1996a; see also Fey, Clegg, \& Fomalont 1996b; Fey \& Charlot 1997) have

TABLE 1

VLBI OBSERVING LOG

\begin{tabular}{|c|c|c|c|c|c|c|}
\hline Epoch & $\begin{array}{c}\lambda \\
(\mathrm{cm})\end{array}$ & $\begin{array}{l}\text { Flux Density } \\
\text { (Jy) }\end{array}$ & $\begin{array}{l}\text { On-Source Time } \\
(\mathrm{hr})\end{array}$ & $\begin{array}{c}\text { Beam } \\
(\text { mas } \times \text { mas })\end{array}$ & $\begin{array}{c}I_{\mathrm{rms}} \\
\left(\mathrm{mJy} \mathrm{beam}^{-1}\right)\end{array}$ & Stations $^{c}$ \\
\hline 1992 Jun $8 \ldots \ldots$ & 13 & 0.957 & 8.5 & $11 \times 4.2$ at -2.2 & 1.0 & FD, LA, KP, LA, NL, PT, Ti, Hh \\
\hline 1992 Jul $9 \ldots \ldots \ldots$ & 13 & 2.032 & 6.5 & $11 \times 1.8$ at -4.2 & 3.3 & FD, Hh, LA, KP, Mc, NL, PT, Ro \\
\hline 1992 Aug $6^{\text {d }} \ldots \ldots$ & 13 & 2.527 & 10 & $\ldots$ & $\ldots$ & $\mathrm{KP}, \mathrm{LA}, \mathrm{Nt}, \mathrm{PT}$ \\
\hline
\end{tabular}

${ }^{a}$ Flux densities obtained from the Green Bank Interferometer monitoring program. The GBI monitored sources only at 3.6 and $13 \mathrm{~cm}$; shown are the flux densities from the GBI monitoring program closest to the epoch of observation.

b This is the maximum duration for any one baseline; some baselines were on-source for shorter periods of time.

${ }^{c}$ Only stations for which correlated data were obtained are listed. Legend: PT, VLBA Pie Town; LA, VLBA Los Alamos; KP, VLBA Kitt Peak; NL, VLBA North Liberty; FD, VLBA Fort Davis; Hh, Hartebeestock; Jb, Jodrell Bank; Mc, Bologna; Nt, Noto; Ro, Deep Space Network DSS65; Ti, Deep Space Network DSS43; Wb, Westerbork

${ }^{d}$ Data were too limited to map the 1992 August 6 epoch. 


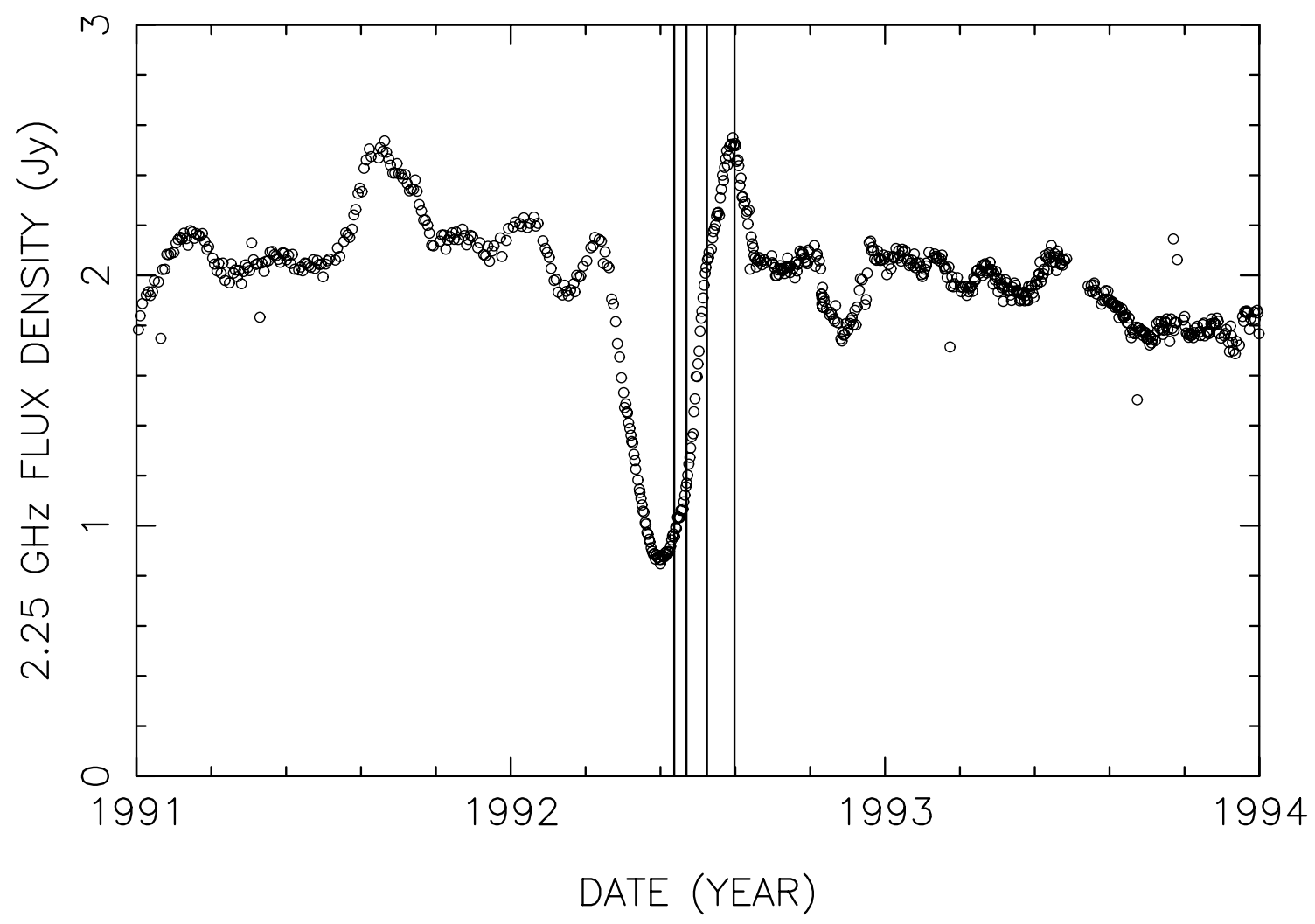

FIG. 1.-Extreme scattering event of PKS $1741-038$. The dots show the $2.2 \mathrm{GHz}(13 \mathrm{~cm})$ flux density as measured by the Green Bank Interferometer. The vertical lines mark the epochs at which the VLBI observations reported in this paper were obtained.

already presented images of PKS $1741-038$ at $6 \mathrm{~cm}$ obtained on 1989 April 12 and at 3.6 and $13 \mathrm{~cm}$ obtained on 1994 July 8. In all images, the source is dominated by a single compact component, and there is little evidence of structural change within the source during the interval 1991-1994. These images have a higher dynamic range than we could obtain from our 1993 June observations. Consequently, we do not include the 1993 June observations here, but instead use the images from the other groups to discuss source characteristics outside the ESE.

Nominal system temperatures for 1992 were obtained from the various stations. These were used for the initial amplitude calibration. A refined amplitude calibration was then determined in the following manner. The source $2121+053$ (OX 036) is a compact source used as a fringe finder during the observing programs. We fitted a single, circular Gaussian to observations of $2121+053$ obtained in $1994 \mathrm{July},{ }^{1}$ finding a diameter of 0.9 mas. With this source structure and the source flux density measured from the GBI monitoring program, we then fitted the $2121+053$ data at each epoch for a single, antenna-based scaling factor. This antenna-based scaling factor accounts for the difference between the assumed and actual system temperatures. Typical corrections were $10 \%-20 \%$.

A crucial assumption, and a potential systematic error, of this method is that the source diameter of $2121+053$ remained essentially constant over the interval 1992-1994. VLBI images of $2121+053$ obtained at epochs bracketing the time of our experiment show it to be extremely compact

\footnotetext{
${ }^{1}$ Observations obtained from the Radio Reference Frame Image Database (RRFID); URL: http://www.usno.navy.mil/RRFID.
}

(Wehrle et al. 1992; RRFID ${ }^{1}$ ). The GBI monitoring program also shows no significant flux-density changes, such as a large increase in the flux density, indicative of structural changes, such as the emergence of a new component. As measured by the GBI, the flux density of $2121+053$ decreased by about $25 \%$ during 1992 , from about $2 \mathrm{Jy}$ to $1.5 \mathrm{Jy}$, as part of a longer term decrease following an outburst in 1988-1989. During 1993 the flux density stopped decreasing, remaining at about $1.5 \mathrm{Jy}$. Superposed upon those longer trends is shorter timescale variability; in the interval 1992 June-August, the flux density varied from near $2 \mathrm{Jy}$ to as low as $1.3 \mathrm{Jy}$, then recovered to $1.7 \mathrm{Jy}$.

Fringe fitting at $13 \mathrm{~cm}$ was performed in two steps. The arrays used consisted of a reasonably close cluster of antennas (mostly in the southwestern US) combined with a smaller number of far-flung antennas. We fitted first for the fringe delays and rates of the antennas comprising the cluster, without solving for the delays and rates of the distant antennas. After applying these delay and rate solutions, we fitted for the delays and rates for the distant antennas only but used all antennas in the fit. This fringefitting procedure increases the probability that we will find fringes to all stations by focusing first on the stations with the highest signal-to-noise ratio and those that should have the smallest rates and delays.

After fringe fitting, a series of first phase-only and then amplitude-and-phase self-calibration iterations were used to account for short timescale fluctuations in antenna gain amplitudes and phases. Because of the extremely compact structure of PKS 1741-038 seen outside the ESE, we often used a point source as the model during phase self- 

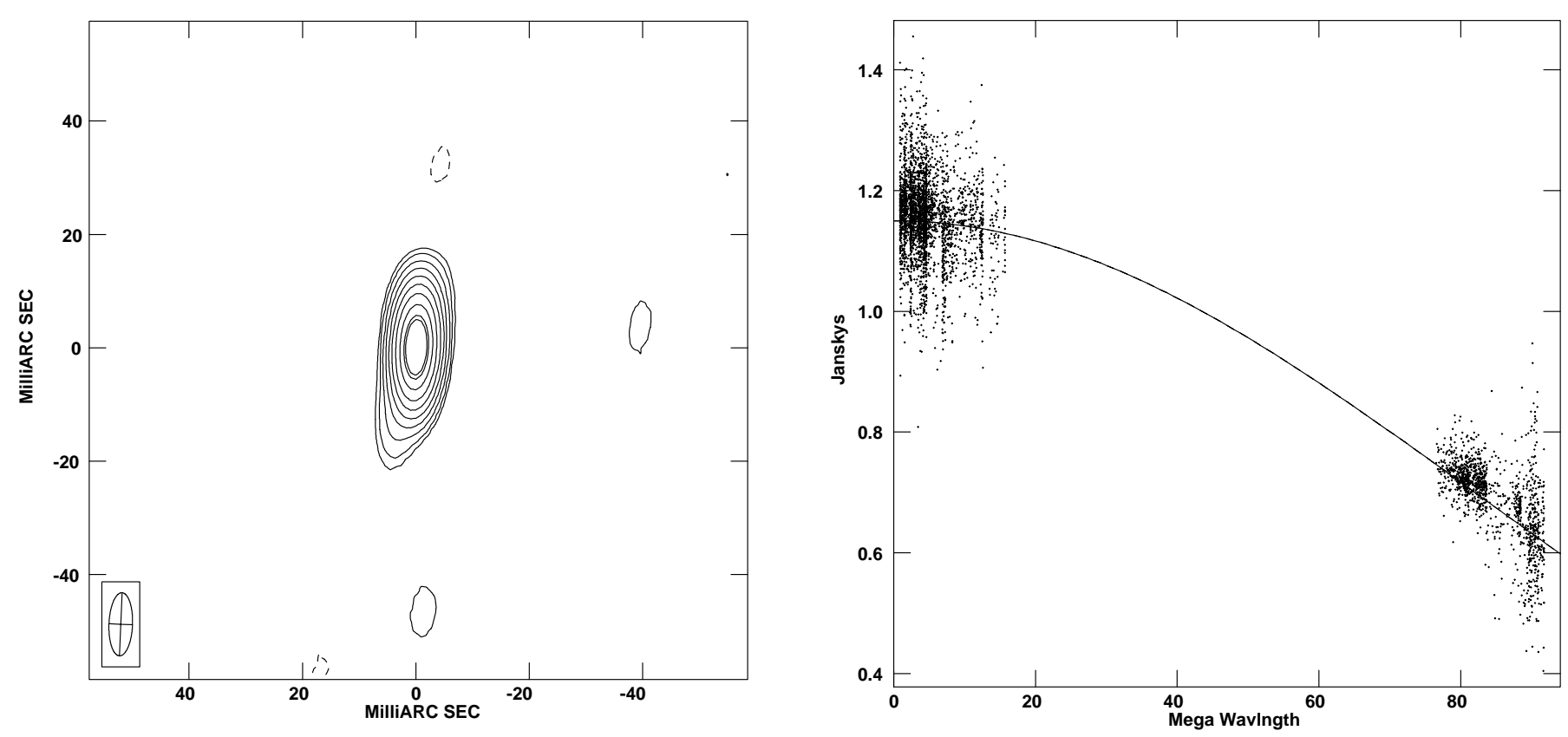

Fig. 2.-Epoch 1992 June 8 at the wavelength $13 \mathrm{~cm}$. Top panel: The image. The off-source noise level is $1 \mathrm{mJy}^{\text {beam }}{ }^{-1}$, and contours are $1 \mathrm{mJy}$ beam $^{-1} \times-3,3,5,10,20, \ldots$ The beam is shown in the lower left. Bottom panel: The visibility data as a function of projected baseline. A model of two components is shown superposed for reference. One component is a circular Gaussian with an amplitude of 1.15 Jy and a diameter equivalent to that implied by the model fit from Table 2, namely 1 mas. The second component is a delta function with an amplitude of 0.01 Jy located 9.7 mas to the south of the first component.

calibration. Since the length of the GBI baseline $(2.4 \mathrm{~km})$ is much shorter than the shortest baseline in our VLBI array (the VLBA_PT-VLBA_LA baseline at $237 \mathrm{~km}$ ), we used the GBI flux densities as zero-spacing flux densities when imaging the source.

Unfortunately, because of either station or correlator problems during the 1992 August 6 observations, only $1 \mathrm{hr}$ of the $10 \mathrm{hr}$ run had more than three stations on-source simultaneously. With only three stations available for most of the run, we can do little more than phase-only selfcalibration and fit simple models to the data. We shall therefore restrict the majority of our comments to the images from the epochs 1992 June 8, June 20, and July 9.

The amount of time on-source ranged between 6 and 10 $\mathrm{hr}$. The resulting thermal noise limit on our maps is therefore approximately $0.5 \mathrm{mJy}_{\text {beam }}{ }^{-1}$. The actual off-source rms noise levels are in the range 1-3 mJy beam ${ }^{-1}$ and are listed in Table 1.

Figures 2, 3, and 4 show the images for the epochs 1992 June 8, June 20, and July 9. We have fit one or more Gaussian components to the $u-v$ data; the solutions to these fits are in Table 2 . We discuss each epoch briefly and separately.

\subsection{June 8 (Fig. 2)}

At this epoch the source is near the minimum flux density. The source structure at this epoch is consistent with that seen outside of the ESE (1992 November, Shen et al. 1997; 1989 April and 1994 July, Fey et al. 1996a), namely, a dominant compact component and a weaker component to the south. We had difficulty finding a model that fitted the data with a nonzero major axis for the secondary component, whereas Fey et al. (1996a) found a major axis of approximately 6 mas. This discrepancy could result from two effects. First, there could have been modest evolution of the secondary component between 1992 and 1994. Such evolution might also account for a portion of the modest source variability seen in the GBI monitoring of PKS $1741-038$. Second, our $u-v$ plane coverage is not as extensive as that of Fey et al. (1996a). Consequently, our coverage may be sufficient to indicate the component's presence without allowing us to fit a detailed model to it.

\subsection{June 20 (Fig. 3)}

The source structure at this epoch consists of a single component. The secondary component is not apparent. Its

TABLE 2

SOURCE MODELs ${ }^{\mathrm{a}}$

\begin{tabular}{cccccccc}
\hline \hline Epoch & $\begin{array}{c}\lambda \\
(\mathrm{cm})\end{array}$ & $\begin{array}{c}S \\
(\mathrm{Jy})\end{array}$ & $\begin{array}{c}r \\
(\mathrm{mas})\end{array}$ & $\begin{array}{c}\psi \\
\left({ }^{\circ}\right)\end{array}$ & $\begin{array}{c}a \\
(\mathrm{mas})\end{array}$ & $\begin{array}{c}b / a \\
\left({ }^{\circ}\right)\end{array}$ & $\phi$ \\
\hline 1992 Jun $8 \ldots \ldots .$. & 13 & 1.15 & 0.0 & 0 & 1.0 & 0.83 & -9.5 \\
& 0.01 & 9.7 & 179 & 0.0 & 1 & $\ldots$ & $\ldots$ \\
1992 Jun $20 \ldots \ldots$ & 18 & 0.95 & 0.0 & 0 & 1.7 & 0.1 & 69 \\
1992 Jul $9 \ldots \ldots$. & 13 & 2.16 & 0.0 & 0 & 0.98 & 0.91 & $\ldots$ \\
1992 Aug $6 \ldots \ldots$. & 13 & 2.52 & 0.0 & 0 & 1.5 & 1 & $\ldots$ \\
\hline
\end{tabular}

${ }^{a}$ Source models consist of Gaussians of flux-density $S$, major axis $a$, and axial ratio $b / a$ at position angle $\phi$ located a distance $r$ from the phase center at a position angle $\psi$. The stronger component was always assumed to be at the phase center. 

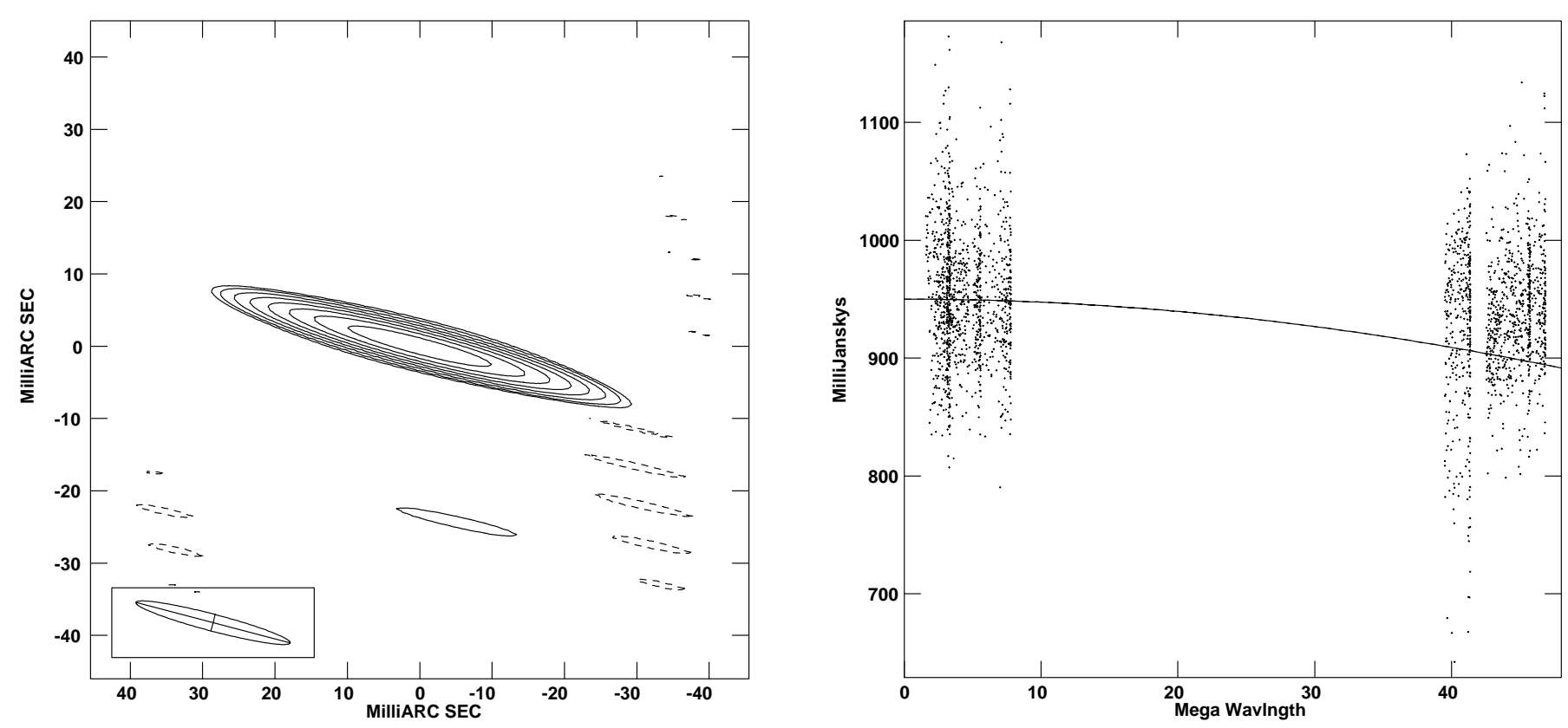

FIG. 3.-Epoch 1992 June 20 at the wavelength $18 \mathrm{~cm}$. Top panel: The image. The off-source noise level is $1.6 \mathrm{mJy}^{-1}$ beam ${ }^{-1}$, and contours are $1.6 \mathrm{mJy}$ beam $^{-1} \times-2,3,5,10,20, \ldots$ The beam is shown in the lower left. Bottom panel: The visibility data as a function of projected baseline. A model of a single, circular Gaussian component is shown superposed for reference. The Gaussian component has an amplitude of 0.95 Jy and a diameter equivalent to that implied by the model fit from Table 2 , namely 0.61 mas.

apparent disappearance is the combination of two effects. First, the observation at this epoch has only four stations, so that detailed structure is likely to be lost. Second, the secondary component has an inverted spectrum, $\alpha \approx 2$ $\left(S_{v} \propto v^{\alpha} ;\right.$ Fey et al. 1996a). Extrapolating this spectrum to 18 $\mathrm{cm}$, we expect that the flux density of the secondary component will be $5-10 \mathrm{mJy}$, sufficiently weak that we would not detect it.

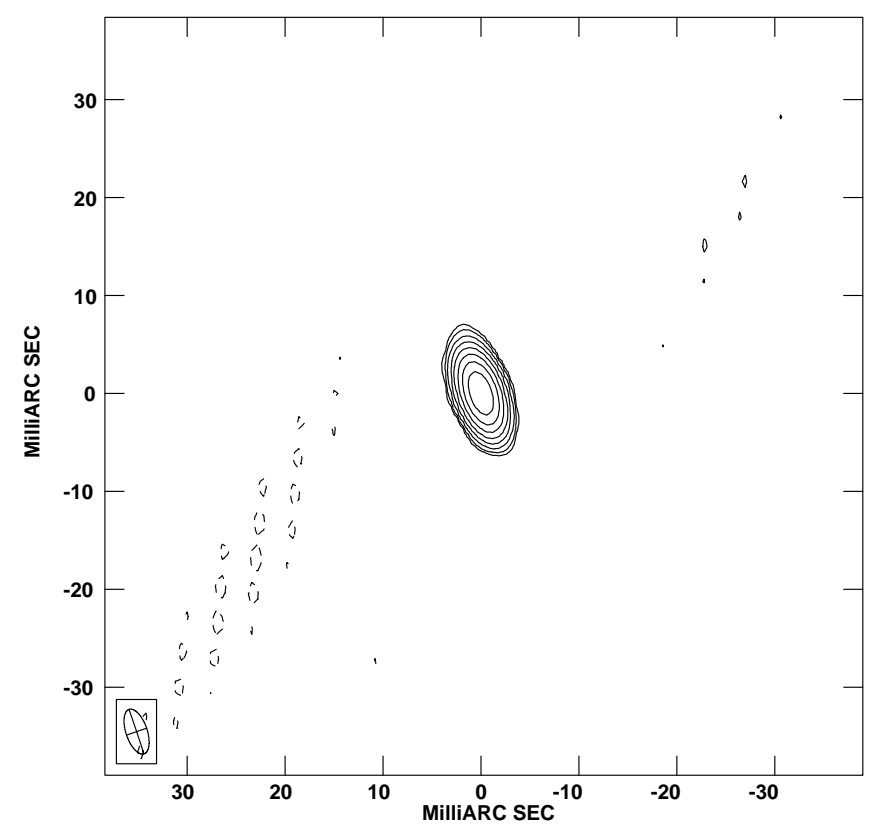

In contrast to the $13 \mathrm{~cm}$ structure, the axial ratio at $18 \mathrm{~cm}$ is $b / a=0.1$. We attach little significance to this low value, however. Because the array contains Hartebeesthoek, it is extended significantly in the north-south direction.

\subsection{July 9 (Fig. 4)}

At this epoch the flux density of the source is starting to return to its nominal value, but this epoch is prior to the

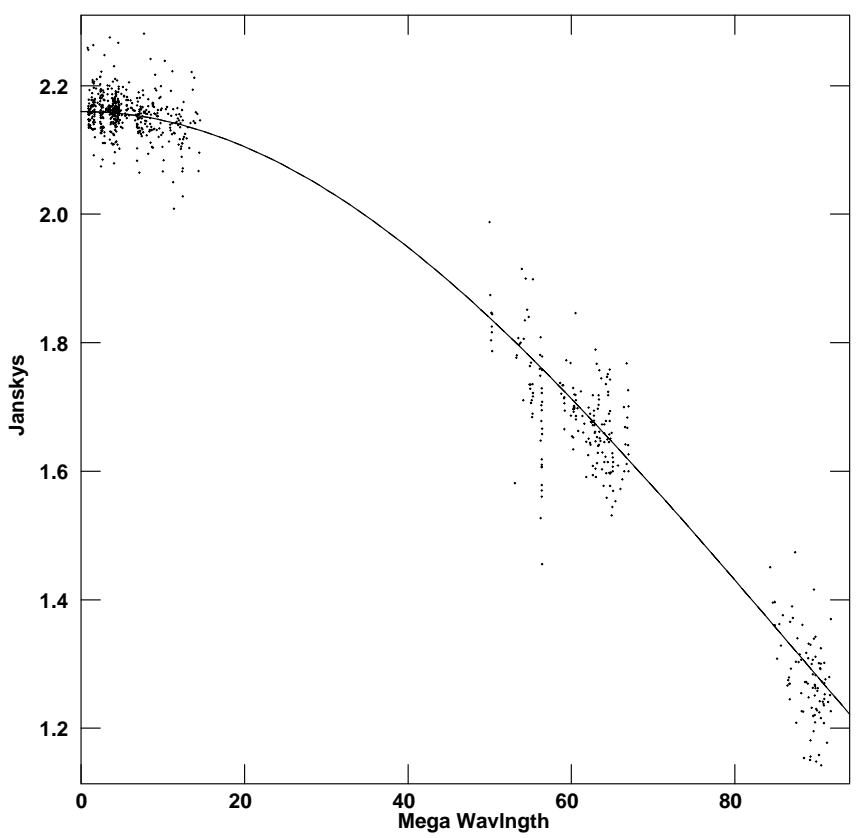

FIG. 4.-Epoch 1992 July 9 at the wavelength $13 \mathrm{~cm}$. Top panel: The image. The off-source noise level is $3.3 \mathrm{mJy}^{\text {beam }}{ }^{-1}$, and contours are $3.3 \mathrm{mJy}$ beam $^{-1} \times-3,3,5,10,20, \ldots$ The beam is shown in the lower left. Bottom panel: The visibility data as a function of projected baseline. A model of a single, circular Gaussian component is shown superposed for reference. The Gaussian component has an amplitude of 2.16 Jy and a diameter equivalent to that implied by the model fit from Table 2, namely 0.93 mas. 
peak flux density during the egress of the ESE. During the ingress and egress from an ESE, when the flux density is above its nominal value, ESE-induced changes in the source structure are most likely to be visible (Clegg et al. 1998). Since we are unable to image the source at its peak flux density (§ 2.4), this epoch represents our best chance for seeing any ESE-induced changes in the source structure.

The source structure is little changed from that outside the event. The source continues to be dominated by a single compact component. The secondary component is not apparent in the image at this epoch. The image at this epoch has the highest off-source noise level of the three epochs. This high noise level is the result of numerous intervals during the observing run in which fringes could not be found. Thus, this observation was more like a series of "snapshots" rather than a pointed observation. The combination of high noise level and poor $u-v$ coverage could contribute to difficulty in detecting the secondary component.

Although limited $u-v$ coverage and the high noise level in this image are at least partially responsible for the absence of the secondary component, another possibility is that the ESE lens did not cover the source fully. Just such a possibility is indicated from Clegg et al.'s (1998) results. They found that a lens comparable in diameter to the compact component, 0.5 mas, produced the best match to the light curve. The relatively simple structure of PKS 1741-038 does not allow us to place any constraints on the axial ratio of the ESE lens, though.

\subsection{August 6}

As noted above, for the majority of this epoch, only three stations were observing the source. Model fitting to the available data indicates that the source continues to have a compact component. The model fitted to the visibility data is listed in Table 2.

\section{DISCUSSION}

In this section we use our VLBI images of PKS $1741-038$ to infer various properties of the lens responsible for this ESE, and in particular, the extent to which refractive and diffractive scattering were important. We begin by showing that the diffractive scattering, as manifested by additional angular broadening of PKS 1741-038 during the event, did occur. Clegg et al. (1998) have modeled the PKS $1741-038$ as due solely to refractive defocusing by an ionized cloud. We discuss the (limited) extent to which our observations can test the predictions of their model. We then turn to the question of the mechanism by which ESEs can occur - refractive defocusing (e.g., Romani et al. 1987; Clegg et al. 1998) or stochastic broadening (Fiedler et al. 1994a). We conclude the section by considering what the shape of our images implies about the shape of the lens and what the line of sight to PKS 1741-038 implies about the genesis of the lens.

\subsection{Diffractive Properties of the PKS 1741-038 ESE Lens}

Our observable for studying the diffractive effects of this lens is the angular broadening of the compact component of PKS $1741-038$. Plasma density fluctuations within the lens will produce angular broadening, in addition to any broadening resulting from density fluctuations along the rest of the line of sight. We shall show that there is a mea- surable amount of angular broadening during the ESE, first by comparing the angular diameter of PKS 1741-038 at different wavelengths during the ESE, then by comparing its angular diameter during the ESE to that after the ESE at the same wavelength. We shall then relate this additional angular broadening to the fluctuations within the lens and show that the lens was probably quite turbulent internally.

The density fluctuations responsible for interstellar scattering (including angular broadening) are typically parameterized by their spatial power spectrum as (Armstrong, Rickett, \& Spangler 1995)

$$
P_{\delta n_{e}}=C_{n}^{2} q^{-\alpha} .
$$

The resulting scattering diameter, for a distant source and assuming $\alpha=11 / 3$ (Rickett 1990), is

$$
\begin{aligned}
\theta_{d} & =128 \mathrm{mas} v_{\mathrm{GHz}}^{-11 / 5} \mathrm{SM}^{3 / 5}, \\
& =1.8 \lambda_{m}^{11 / 5} \mathrm{SM}^{3 / 5},
\end{aligned}
$$

where $v_{\mathrm{GHz}}$ is the observing frequency in $\mathrm{GHz}, \lambda_{m}$ is the observing wavelength in meters, and

$$
\mathrm{SM}=\int C_{n}^{2}(z) d z .
$$

In the local ISM $C_{n}^{2} \sim 10^{-3.5} \mathrm{~m}^{-20 / 3}$ (Armstrong et al. 1995), and $\mathrm{SM} \sim 10^{-3.5} \mathrm{kpc} \mathrm{m}^{-20 / 3}$ for a typical $1 \mathrm{kpc}$ path length through the local ISM.

During the ESE we find $13 \mathrm{~cm}$ diameters of 0.8 and 1 mas and an $18 \mathrm{~cm}$ diameter of 1.6 mas (Table 2). Taking the average of the $13 \mathrm{~cm}$ diameters, we find the angular diameter scales as $\theta \propto \lambda^{1.8}$. We regard this wavelength scaling as consistent with that expected for angular broadening, but not decisive evidence in favor of it. We have not attempted to correct for any contribution by intrinsic structure, which should have a weaker wavelength dependence than angular broadening. Furthermore, some deviation from a strict $\lambda^{2.2}$ dependence might be expected because we are comparing angular diameters determined at different epochs during a time-dependent event. Indeed, the angular diameter at 13 $\mathrm{cm}$ does change in exactly the manner expected if the lens is centrally condensed; near the flux-density minimum (1992 June 6 epoch) when the path length through a centrally condensed lens would be near the maximum, the angular diameter is slightly larger (1 mas) as compared to the angular diameter (0.8 mas) near the end of the ESE (1992 July 9), when the path length through the lens would be shorter. However, our determination of the $\lambda^{1.8}$ scaling does depend crucially upon the $18 \mathrm{~cm}$ diameter, and, in turn, on the limited $u-v$ coverage for this observation. If we fit a circular Gaussian instead of an elliptical Gaussian to the 18 $\mathrm{cm}$ visibility data, we find an angular diameter of 0.3 mas, implying a $\lambda^{-3.4}$ dependence. Hence, we regard the comparison of angular diameters during the ESE as suggestive, but not compelling, evidence, for increased angular broadening during the ESE.

We find a more compelling demonstration of an increase in the angular diameter from comparing the angular diameter of PKS 1741-038 during and after the ESE. During the ESE the $13 \mathrm{~cm}$ diameter of PKS 1741-038 was $0.9 \pm 0.1$ mas. In a series of subsequent observations, during 1994-1997 (Fey et al. 1996a, 1996b; Fey \& Charlot 1997; A. L. Fey, unpublished data), acquired while the source was not undergoing an ESE, the angular diameter of the compact component has been measured to be $0.5-0.75$ 
mas, with a mean of $0.63 \pm 0.04$ mas. The quoted uncertainty in the mean diameters are statistical. Systematic effects, namely the length of the longest baseline in the VLBI array used, probably contribute to an overestimation of the diameter. The same systematic effects appear to contribute to our fits also being overestimates of the actual diameter. Time-dependent changes may also affect the diameters during the ESE.

We find the excess angular broadening due to the ESE lens by subtracting in quadrature the diameters during and after the ESE. Using the diameters determined above, namely $\theta_{\text {in }}=0.9$ mas and $\theta_{\text {out }}=0.63$ mas, we find $\delta \theta_{d} \equiv$ $\left(\theta_{\text {in }}^{2}-\theta_{\text {out }}^{2}\right)^{1 / 2} \lesssim 0.7$ mas. We treat this value as an upper limit based on our assessment of the systematic uncertainties in the measured diameters. However, our longest baseline is often significantly longer than that used in determining the diameters after the ESE, so our measurement of an increase in the diameter of the source is robust.

The excess angular broadening of the lens arises from an additional $C_{n}^{2}$ in the line of sight to PKS 1741-038 during the ESE. We solve for the level of scattering within the lens, $\mathrm{SM}_{\text {lens }}$, in the following manner. The scattering within the lens is given by

$$
\mathrm{SM}_{\text {lens }} \equiv \mathrm{SM}_{\mathrm{in}}-\mathrm{SM}_{\text {out }} \text {, }
$$

where $\mathbf{S M}_{\mathrm{in}}$ and $\mathbf{S M}_{\text {out }}$ are the scattering measures seen inside and outside the ESE, respectively.

We have estimated SM $_{\text {out }}$ from RRFID observations (Fey et al. 1996a; Fey et al. 1996b; Fey \& Charlot 1997; Fey, unpublished data). The RRFID observations acquire 3.6 and $13 \mathrm{~cm}$ data simultaneously. We have fit the measured 3.6 and $13 \mathrm{~cm}$ diameters to

$$
\theta^{2}(\lambda)=\theta_{d, 1}^{2} \lambda_{m}^{4.4}+\theta_{I, 1}^{2} \lambda_{m}^{2},
$$

assuming that the scattering and intrinsic diameters add in quadrature and that the intrinsic diameter scales as $\lambda^{1}$, as is appropriate for a synchrotron self-absorbed component (Kellerman \& Owen 1988). Here $\theta_{d, 1}$ and $\theta_{I, 1}$ are the scattering and intrinsic diameters, respectively, at the fiducial wavelength of $1 \mathrm{~m}$. The spectral index of PKS $1741-038$ is $\alpha \approx 0.17\left(\mathrm{~S} \propto v^{\alpha}\right)$ around the time of the ESE, but excluding the ESE itself, and $\alpha \approx 0.37$ near the time of the RRFID observations, in both cases consistent with the assumption of self-absorption. We then use equation (2) to solve for $\mathrm{SM}_{\text {out }}$. We find $\mathrm{SM}_{\text {out }}=10^{-3} \mathrm{kpc} \mathrm{m}^{-20 / 3}$.

This value of $\mathrm{SM}_{\text {out }}$ is consistent with the available constraints on the scattering diameter of PKS 1741-038 from low frequencies. Using interplanetary scintillation observations at $92 \mathrm{~cm}$, Vijayanarasimha et al. (1985) determined that PKS 1741-038 must have a component with a diameter of 100 mas. Assuming that interstellar scattering dominates at this wavelength, we find a scattering measure $\mathrm{SM}_{\text {out }} \leq 10^{-2} \mathrm{kpc} \mathrm{m}^{-20 / 3}$. We treat this value as an upper limit because IPS observations do not give detailed information on the source structure.

We estimate $\mathbf{S M}_{\mathrm{in}}$ from the difference in the diameter of PKS 1741-038 inside and outside of the ESE,

$$
\left(\delta \theta_{d}\right)^{2}=\theta_{\mathrm{in}}^{2}-\theta_{\text {out }}^{2}=\left(1^{\prime \prime} .8\right)^{2} \lambda_{m}^{22 / 5}\left(\mathbf{S M}_{\mathrm{in}}^{6 / 5}-\mathbf{S M}_{\text {out }}^{6 / 5}\right) .
$$

We have already constrained $\delta \theta_{d} \leq 0.7$ mas. We find $\mathrm{SM}_{\text {in }} \sim 10^{-2.4} \mathrm{kpc} \mathrm{m}^{-20 / 3}$, a value of SM that is somewhat larger than the typical SM through the local ISM.

Thus, the ESE lens toward PKS 1741-038 produced an additional angular broadening of approximately 0.7 mas, and the lens itself had a scattering measure of $\mathrm{SM}_{\mathrm{lens}}=$ $10^{-2.5} \mathrm{kpc} \mathrm{m}^{-20 / 3}$.

In order to produce a significant flux density suppression during the ESE, from stochastic broadening alone, the angular diameter of the lens must be comparable to that of the source (Fiedler et al. 1994a). We assume that the lens diameter was $a \sim 1$ mas (§3.3) or $a \sim 0.1 \mathrm{AU}(D / 0.1 \mathrm{kpc})$, where $D$ is the distance to the lens. Because the extent of the lens along the line of sight may be (considerably) different than its transverse size, we take $\mathrm{SM}_{\text {lens }}=C_{n \text {,lens }}^{2} \eta a$, where the factor $\eta$ is the ratio of the lens's extent along the line of sight to its transverse extent. We find $C_{n, \text { lens }}^{2} \sim 10^{7} \eta^{-1}$ $\mathrm{m}^{-20 / 3}(D / 0.1 \mathrm{kpc})^{-1}$. For comparison, Hjellming \& Narayan (1986) estimated that $C_{n}^{2} \gtrsim 10^{-1.5} \mathrm{~m}^{-20 / 3}$ for this line of sight using the refractive scintillation of this source outside of the ESE.

One of the key features of ESE-like events observed toward pulsars is that the pulse width does not increase (Cognard et al. 1993; Lestrade et al. 1998; Maitia et al. 1998). Using our estimate of $\mathbf{S M}_{\text {lens }}$, we can predict how much pulse broadening, another diffractive effect, would be produced from a lens comparable to that which passed in front of PKS 1741-038. The amount of pulse broadening is (Taylor \& Cordes 1993)

$$
\tau_{d} \leq 1.1 \mathrm{~ms} D_{\mathrm{kpc}}(\mathrm{SM})^{6 / 5} v_{\mathrm{GHz}}^{-22 / 5} .
$$

We can calculate only an upper limit because the amount of pulse broadening depends upon the location of the lens along the line of sight (Cordes \& Rickett 1998); the maximum occurs when the lens is midway between the observer and pulsar. We predict that ESE lenses typical of the one that passed in front of PKS 1741-038 will increase the pulse broadening of a background pulsar by only $1.1 D_{\mathrm{kpc}} \mu \mathrm{s}$ at $1 \mathrm{GHz}$. This small amount is consistent with the lack of broadening seen toward the millisecond pulsars PSR B1937+ 21 and PSR J1643 - 1224.

Our analysis has assumed that scattering within this lens can be described in terms of a power-law spectrum of density fluctuations (eq. [1]). While this may be true within the lens (\$ 3.1.1), the lens itself cannot be formed by the same processes that give rise to the spectrum of density fluctuations in the local ISM (Armstrong et al. 1995). First, a medium pervaded by density fluctuations on AU scales should produce a correlation between flux density and angular diameter (Blandford \& Narayan 1985, their Figs. 1 and 4). In contrast, we observe an anticorrelation, with the angular diameter increasing as the flux density decreases. Second, if the values we have used for $a$ and $D$ are not severe underestimates, the large value of $C_{n \text {, lens }}^{2}$ we infer is significantly larger than the value in the local ISM. Even allowing for a structure extremely elongated along the line of sight, $\eta>100, C_{n, \text { lens }}$ remains orders of magnitude above the value in the local ISM. We regard the large value of $C_{n, \text { lens }}^{2}$ as an indication that the genesis of an ESE lens requires an energetic process.

\subsubsection{The Electron Density Power Spectrum within an ESE Lens}

The measured visibility on an interferometer baseline $b$ is

$$
V(b)=e^{-D_{\phi}(b) / 2},
$$

for a point source seen through a region of density fluctuations with a spatial power spectrum given by equation (1). The phase structure function $D_{\phi}(b)$ is a measure of the fluctuations induced in the wavefront's phase as it propagates 
through the scattering medium and is given by (e.g., Cordes \& Lazio 1991)

$$
D_{\phi}(b) \propto \theta_{d}^{\beta} b^{\beta} .
$$

Here $\theta_{d}$ is the diffractive scattering angle, and $\beta \equiv \alpha-2$.

There are a number of lines of sight that suggest $\alpha \approx 11 / 3$, the Kolmogorov value (Rickett 1990). There are also some lines of sight that suggest a significantly larger value, $\alpha>4$. The diameters tabulated in Table 2 were found by fitting a Gaussian to the visibilities, i.e., assuming $\beta=2$. We now relax that requirement.

For the three epochs for which we were able to produce images, we fitted the visibility data with a model of the form of equation (8). For all three epochs we were unable to place any meaningful constraints on $\beta$. Allowed values of $\beta$, at all three epochs, were $1-2.2$. Though the range is nearly centered on the Kolmogorov value of 1.67 , we cannot exclude $\beta \geq 2$.

\subsection{Refractive Properties of the PKS 1741-038 ESE Lens}

Refractive effects expected from ESE lenses include substructure within the source, angular position wander, and multiple imaging, though the extent to which any of these occur depends upon the strength of refraction within the lens. There is no indication of refractively induced substructure in the source such as might be produced if the lens had substructure within it. During the event, the source consisted of a compact component, with a weak secondary component detectable to the south in at least one epoch. This structure is essentially identical to that seen after the event (Shen et al. 1997; Fey et al. 1996a). Below, we quantify the possibility that the shape of the lens has altered the shape of the source (\$ 3.4).

One of the key predictions of a refractive model for ESEs is that an ESE should produce angular position wander of the background source. Clegg et al. (1998) predicted that during the PKS 1741-038 ESE, the angular position of the source wandered by 0.4 mas at $13 \mathrm{~cm}$ and by 0.8 mas at 18 $\mathrm{cm}$. The observations reported here were not phasereferenced, and the self-calibration we performed erased all absolute position information. Furthermore, the proximity of the two intrinsic components of PKS 1741-038, the sparse visibility data, and limited dynamic range (particularly of the 1992 July 9 observations) give us little confidence of detecting relative position shifts between the two components, such as would occur if the lens covered only one component at a time. Consequently, even if the lens covered only the brighter component, it would be difficult to determine the relative separation, with any degree of confidence, between the two components in either the image or visibility domains. We are thus unable to test the prediction of ESE-induced angular position wander.

A second prediction is that an ESE can produce multiple imaging of the background source. In the case of this ESE, any secondary image(s) must have been either extremely faint or only slightly offset with respect to the primary image. Within the $10 \%-15 \%$ uncertainties of the GBImeasured flux densities (Fiedler et al. 1987b) and those in our amplitude calibration, which we estimate to be at least $10 \%$, our models account for all of the flux density measured by the GBI. A bright secondary image(s) could have been present only if the multiple images nearly overlapped so that no significant anisotropy was produced in our VLBI images (Table 2). Clegg et al. (1998) predicted that this ESE was not strong enough to form caustics and produce multiple imaging.

\subsection{The ESE Mechanism}

As noted in $\S 1$, two general classes of models have been advanced to explain how ESEs occur. In the refractive defocusing model (Romani et al. 1987; Clegg et al. 1998; Walker \& Wardle 1998; hereafter, the RD model) the decrease in the source's flux density during the event occurs because of refractive defocusing of the incident rays on the lens. In the stochastic broadening model (Fiedler et al. 1994a; hereafter, the SB model) the flux density decrease occurs because small-scale inhomogeneities in the lens scatter the incident wave front. While most recent work has focussed on the RD model - particularly the work of Clegg et al. (1998), who compared quantitatively the observed light curve and that predicted from an RD model - both models can reproduce the generic features of an ESE light curve, and the only other observational data with which to compare the models have been the pulsar timing programs described in $\S 1$.

A key prediction of the refractive model is that the source's flux density and angular diameter should be highly correlated. In contrast we observe an anticorrelation, more consistent with that expected from the SB model. Fey et al. (1996b) discussed qualitatively how the SB model could produce the PKS 1741-038 ESE, but did not compare quantitatively the observed light curve and the SB model. Consequently, we shall revisit the question of the mechanism by which ESEs are produced and whether the SB model can account for this ESE.

We shall use the SB model developed by Fiedler et al. (1994, Appendix A). This model describes the flux density of a source during an ESE as $\hat{F}\left(t ; F_{0}, \mu, \theta_{I}, \theta_{l}, \theta_{b}\right)$. Here $F_{0}$ is the source's nominal flux density outside the lens, $\mu$ is the proper motion of the lens across the lie of sight, $\theta_{I}$ is the intrinsic (FWHM) angular diameter of the background source, $\theta_{l}$ is the apparent angular width of the lens, and radiation incident on the lens is scatter broadened by $\theta_{b}$ (FWHM).

We used a grid-search method to search the available parameter space, evaluating the goodness of fit by the $\chi^{2}$ statistic. Based on the measurements of PKS 1741-038 outside the ESE and our determination of the additional angular broadening during the event (§3.1), we began by holding $\theta_{I}$ and $\theta_{b}$ fixed at $\theta_{I}=0.5$ mas and $\theta_{b}=0.7$ mas. We thus fit for the parameters $F_{0}, \mu$, and $\theta_{l}$.

We were unable to find reasonable agreement. In particular the best-fit model had a flux-density minimum that was approximately $85 \%$ of the nominal flux density as opposed to the $50 \%$ minimum that was observed. Furthermore, the shape of the modeled ESE light curve is that of a flatbottomed minimum rather than the rounded minimum observed. We then removed the constraints on $\theta_{I}$ and $\theta_{b}$ separately. If we fit for $\theta_{I}, F_{0}, \mu$, and $\theta_{l}$ while holding $\theta_{b}$ fixed, the fit agreement improves slightly. However, the depth of the modeled flux-density minimum continues to be insufficient to match the observed depth, and the minimum is flat-bottomed as opposed to the observed rounded minimum.

If we fit for $\theta_{b}, F_{0}, \mu$, and $\theta_{l}$ while holding $\theta_{I}$ fixed, we find quite reasonable agreement with both the depth and shape of the minimum being reproduced. Table 3 lists our best fitting parameters. The significant result of our fit is that the broadening diameter required to reproduce the observed 
TABLE 3

\begin{tabular}{|c|c|}
\hline \multicolumn{2}{|c|}{$\begin{array}{c}\text { PARAMETERS OF THE BeST-Fit } \\
\text { StOCHASTIC BROADENING } \\
\text { MODEL }\end{array}$} \\
\hline Parameter & Value \\
\hline$\theta_{I}^{\mathrm{a}}$ (mas) $\ldots$ & 0.5 \\
\hline$\theta_{b}$ (mas) $\ldots . .$. & 2 \\
\hline$F_{0}(\mathrm{Jy}) \ldots \ldots$ & 1.9 \\
\hline$\theta_{l}$ (mas) $\ldots \ldots \ldots \ldots$ & 1 \\
\hline$\mu\left(\mu\right.$ as $\left.\operatorname{deg}^{-1}\right) \ldots \ldots$ & 14 \\
\hline$\chi^{2} \ldots \ldots \ldots \ldots \ldots \ldots$ & 16 \\
\hline
\end{tabular}

a This parameter held fixed.

light curve is much larger than what we infer from our measurements. The SB model alone requires a broadening angle $\theta_{b}=2$ mas, while our measurements suggest that only an additional 0.7 mas of broadening occurred during the event.

Moreover, the value of $\theta_{b}$ found is probably a lower limit. The model of Fiedler et al. (1994a) assumes that $\theta_{b}$ is constant across the width of the lens. If the strength of broadening varies across the lens, being stronger in the center and weaker around the edges, an even larger value of $\theta_{b}$ would be required to obtain the same decrease in flux density during the event.

We therefore conclude that the SB model alone cannot explain both the observed light curve and amount of angular broadening. Given the good agreement that Clegg et al. (1998) found using the RD model, we consider it likely that both refractive defocusing and stochastic broadening are occurring.

\subsection{Image Anisotropy}

The images shown in Figures 2-4 display varying degrees of anisotropy. We can identify six causes of image anisotropy (Cordes et al. 19876; Cordes 1990; see also Spangler \& Cordes 1988): (1) incomplete $u-v$ coverage; (2) intrinsic source structure; (3) anisotropic diffractive scattering; (4) anisotropic refractive focusing; (5) unresolved multiple images; and (6) a spatially limited scattering volume.

The first cause - incomplete $u-v$ coverage - is almost certainly the explanation for the image of 1992 June 20. The array was elongated significantly in the north-south direction because it included Hartebeestock. The $u-v$ coverage was considerably more uniform for the other two epochs, though, and incomplete $u-v$ coverage is less likely to be the cause of the image anisotropy at these epochs.

While we cannot rule out the possibility of one or more of the remaining causes contributing to the source anisotropy, we can set limits on the shape of the lens by assuming all of the image anisotropy is due to refractive focusing. The electron density profile across the lens produces a refractive gain $G$. If the density profile in two orthogonal directions differs, the source will have an axial ratio (Cordes et al. 1986; Spangler \& Cordes 1988)

$$
\frac{b}{a}=\frac{G_{b}}{G_{a}} .
$$

The axial ratios we measure during the ESE are $b$ / $a>0.8$. These axial ratios are much closer to unity than would be expected if the lens had an axial ratio $\eta \sim 100$ (e.g., Romani et al. 1987), and its long axis was in or near the plane of the sky. We conclude that the refractive strength of the ESE lens was not considerably different in different directions on the plane of the sky.

Romani et al. (1987) have speculated that ESE lenses are filamentary structures. Filamentary structures have the desirable property of reducing the degree to which the ESE lenses are overpressured with respect to the nominal interstellar pressure. As we discuss above, filamentary structures would also reduce the inferred level of turbulence within the lenses. Filamentary ionized structures would also form naturally in a magnetized medium. If ESE lenses are filamentary structures, the nearly isotropic image shapes that we observe indicate that the lenses are extended along the line of sight. Lestrade et al. (1998) have suggested that ESEs toward pulsars occur only when filamentary or sheetlike structures are favorably oriented along the line of sight. Our VLBI images suggest that a similar situation is necessary for ESEs toward extragalactic sources.

\subsection{Environments of ESE Lenses}

Fiedler et al. (1994b) showed that the lines of sight to a small number of sources that have undergone an ESE display structures suggestive of a turbulent origin. For instance, the line of sight to $0954+658$ passes near the edge of radio Loop III, with filamentary structures seen in 100 $\mu \mathrm{m}$ emission, and the line of sight to $2352+495$ (DA 611) passes near RAFGL 5797S, an infrared source with a cometary morphology. Fiedler et al. (1994a) argued that the distribution of ESE sources near the edges of these radio loops was not accidental but was indicative of a connection between ESEs and sites of interstellar turbulence such as old supernova remnants. Supernova remnants could also provide a high-pressure environment in which the ESE lenses could survive (Romani et al. 1987). Walker \& Wardle (1998) have since suggested that ESEs arise from dense molecular clouds in the Galaxy's halo.

The line of sight to PKS 1741-038 displays similar evidence of strong gradients and turbulent-like structures. Figure $5 a$ shows the $\mathrm{H}$ I column density toward the line of sight of PKS $1741-038$ and 1749+ 096 (4C 09.57), another source observed to have undergone an ESE. The H I column density displays a strong gradient with Galactic latitude, changing by a factor of nearly $10^{2}$ over $10^{\circ}$ in latitude. Furthermore, the two spurs of $\mathrm{H}$ I emission extending to higher latitudes in Figure $5 a$ are part of radio Loop I, a structure Fiedler et al. (1994a) have already suggested is responsible for the ESE toward 1749+096. Like 1749+096, PKS $1741-038$ is close to, perhaps within, a portion of Loop I. Figure $5 b$ shows the $100 \mu \mathrm{m}$ emission toward PKS 1741-038. While not as dramatic as the structures seen along the lines of sight toward $0954+658$ and $2352+495$, PKS 1741-038 does appear near a local minimum in the $100 \mu \mathrm{m}$ emission. The appearance of the emission is suggestive of a process that has excavated a cavity in the ISM. Ionized gas, with significant density enhancements, could result within the cavity or on its edges.

We cannot conclude, from these $\mathrm{H}$ I and $100 \mu \mathrm{m}$ images alone, that ESEs are produced at sites of interstellar turbulence, but these images do add to the existing circumstantial evidence suggesting that this is the case.

It might also be possible to place further constraints on the distance to the material responsible for ESEs in this direction. The pulsar PSR J1743 - 0337 (PSR B1740 - 03) is located only $18^{\prime}$ away from PKS $1741-038$. It has a disper- 


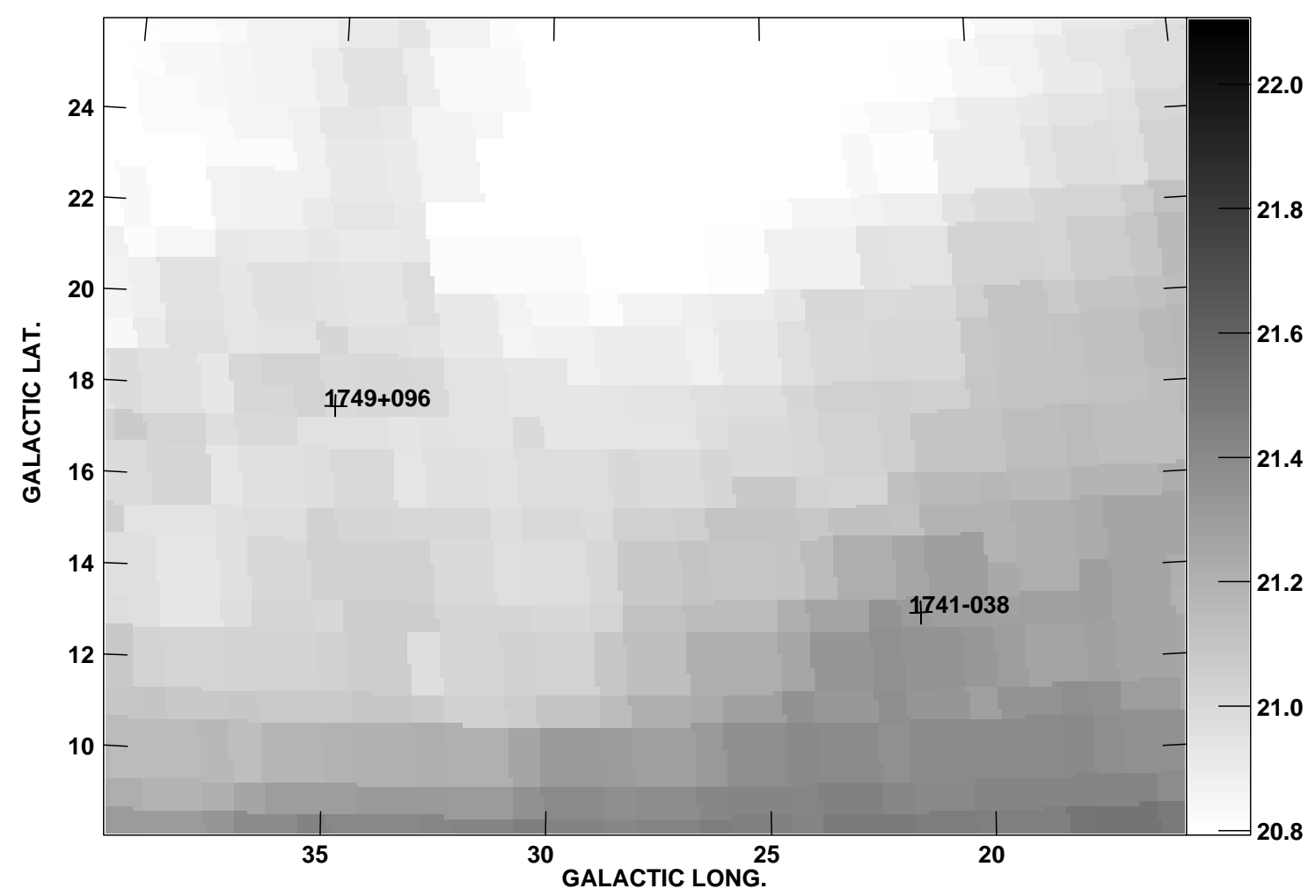

Fig. $5 a$

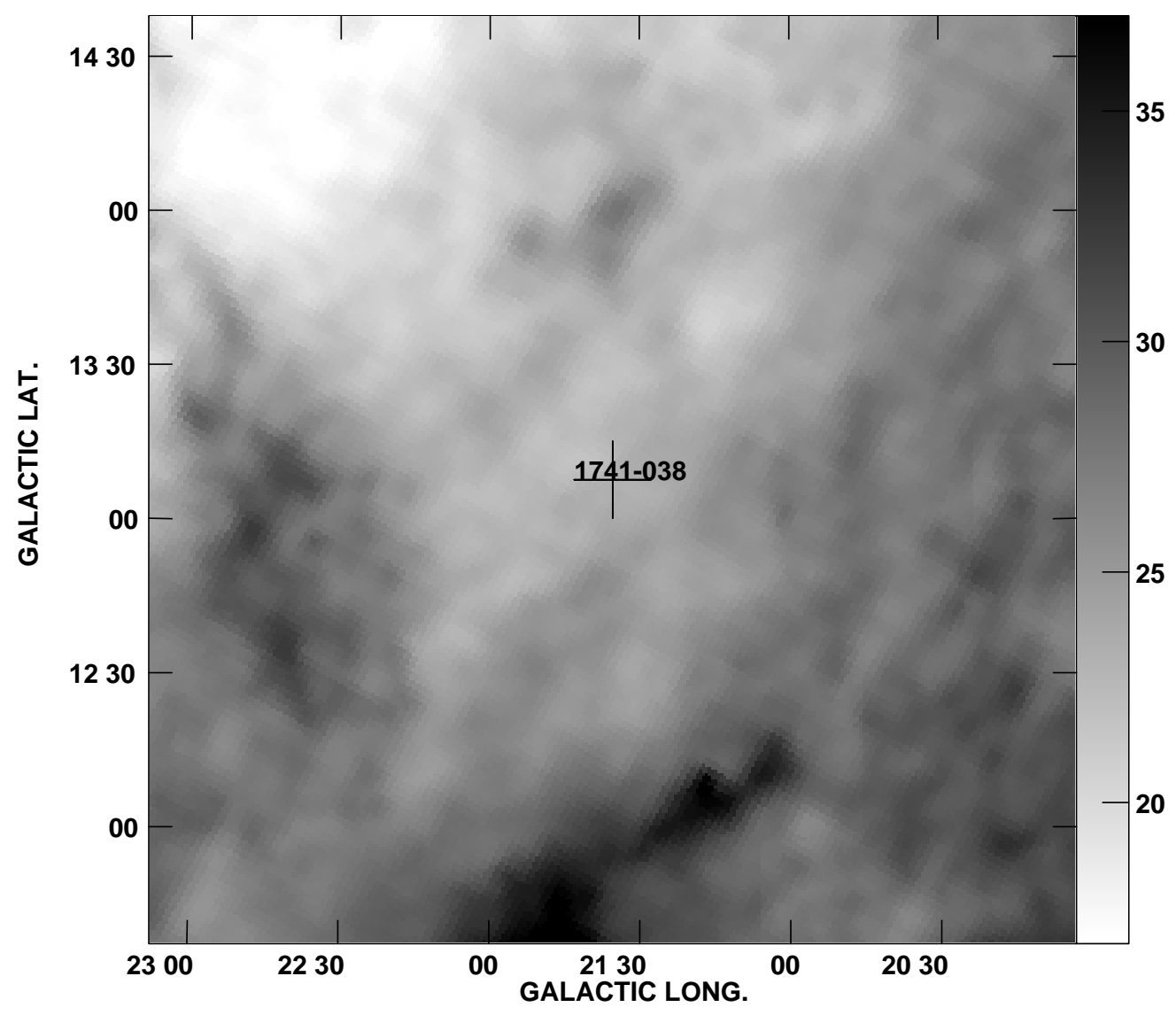

Fig. $5 b$

FIG. 5.-Environs of PKS 1741-038. (a) The gray scale shows the column density of H I (Dickey \& Lockman 1990$)$, ranging between $10^{20.8} \mathrm{~cm}^{-2}($ white) and $10^{22.1} \mathrm{~cm}^{-2}$ (black). The positions of PKS 1741-038 and 1749+096, another source observed to have undergone an ESE, are marked. (b) The gray scale shows the $100 \mu \mathrm{m}$ emission, as derived from IRAS observations, ranging between $17 \mathrm{MJy} \mathrm{sr}^{-1}$ (white) and $37 \mathrm{MJy}^{-1}$ (black). The position of PKS $1741-038$ is marked. 
sion measure of $35 \mathrm{pc} \mathrm{cm}^{-3}$, corresponding to a distance estimate of $1.8 \mathrm{kpc}$ (Taylor \& Cordes 1993). If this pulsar showed enhanced scattering or refractive events, like ESEs or fringing in a dynamic spectrum, that would be a strong indication that at least a portion of the scattering in this direction occurs because of material closer than $1.8 \mathrm{kpc}$.

\section{CONCLUSIONS}

We have presented the first VLBI images of a source, PKS 1741-038, obtained at multiple epochs as the source underwent an extreme scattering event.

We have used these images to assess two models for the origin of ESEs - a refractive defocusing model and a stochastic broadening model. The source structure is dominated by a compact component and is essentially unchanged during the event as compared to the structure after the event. The only change is a slight increase in the diameter of the source (by $0.7 \mathrm{mas}$ ), an increase we attribute to additional angular broadening within the lens. This additional angular broadening is consistent with that expected from a stochastic broadening model but is not consistent with that expected from a purely refractive defocusing model. Specifically, a refractive defocusing model predicts a correlation between the flux density and angular diameter of the source. However, attempts to reproduce the ESE light curve of PKS $1741-038$ by a purely SB model require a larger increase in the source's diameter $(2 \mathrm{mas})$ than is observed $(0.7$ mas). We cannot test other predictions of the RD model. The refractive defocusing model predicts angular position wander of the source, but our observations were not sensitive to absolute angular position shifts. We also see no evidence of strong multiple imaging, but a quantitative comparison of the ESE light curve and an RD model predicts that the refractive strength of the lens was not sufficient to produce multiple imaging (Clegg et al. 1998). We conclude that the PKS 1741-038 ESE involved both SB and RD processes.

The angular diameter of PKS 1741-038 increased by about 0.7 mas during the ESE. The amount of angular broadening contributed by the lens implies that the interiors of lenses are highly turbulent with levels of scattering orders of magnitude higher than that seen in the local ISM. The inferred level of the electron density power spectrum, as parameterized by the coefficient $C_{n}^{2}$, is $C_{n \text {, lens }}^{2} \sim 10^{7} \eta^{-1}$ $\mathrm{m}^{-20 / 3}(D / 0.1 \mathrm{kpc})^{-1}$. A filamentary lens, with $\eta>1$, would decrease the required $C_{n}^{2}$.

The observed visibility data are consistent with the interior of the lens having a power-law density power spectrum, with a power spectral index similar to that seen in the local ISM, though we cannot rule out a "steep" density spectrum $(\beta>4)$. The lens itself could not have arisen from the density fluctuations in the local ISM, however. The value of $C_{n, \text { lens }}^{2}$ is well in excess of the local value, and evenly distributed density fluctuations would give rise to a flux densityangular diameter correlation in contrast to the observed anticorrelation.

If ESE lenses are filamentary, as has been suggested to reduce their overpressure relative to the ambient medium, ESEs must occur only when the filamentary structures are seen nearly lengthwise. A filamentary lens seen transverse to its long axis would produce different refractive gains along and across the lens, resulting in image anisotropy. Our images display little anisotropy.

The line of sight toward PKS $1741-038$ shows a strong gradient in the neutral hydrogen density, and the source lies close to or within radio Loop I, with small-scale, "cavity"like structure seen at $100 \mu \mathrm{m}$. Such interstellar structures along the line of sight to PKS 1741-038 are similar to that seen toward some of the other sources for which ESEs have been observed. This line of sight is thus consistent with ESE lenses originating from energetic turbulent processes in the ISM.

Future observations of a source undergoing an ESE will be enhanced by the existence of dedicated VLBI arrays such as the Very Long Baseline Array. A key prediction of the refractive model - one that we have been unable to test - is the existence of angular wandering. Future observations should also have a more extensive and uniform $u-v$ plane coverage, making the imaging process easier. Modern, frequency-agile receivers also allow for the possibility of simultaneous or nearly simultaneous images at multiple frequencies. Observations at multiple frequencies should include imaging the source in the $\mathrm{H}$ I line to search for the existence of neutral structures related to the ionized structures responsible for ESEs. The major impediment to a set of such observations is the lack of a existing monitoring program that could find additional ESEs.

We thank S. Spangler for helpful discussions regarding interstellar refraction, and K. Desai for his image-fitting software. This research made use of NASA's Astrophysics Data System Abstract Service; the SIMBAD database, operated at the CDS, Strasbourg, France; and NASA's SkyView facility ${ }^{2}$ located at NASA Goddard Space Flight Center. A portion of this work was performed while T. J. W. L. held a National Research Council-NRL Research Associateship. Basic research in radio astronomy at the NRL is supported by the Office of Naval Research.

${ }^{2}$ URL: http://skyview.gsfc.nasa.gov/

\section{REFERENCES}

Armstrong, J. W., Rickett, B. J., \& Spangler, S. R. 1995, ApJ, 443, 209

Blandford, R., \& Narayan, R. 1985, MNRAS, 213, 591

Clegg, A. W., Fey, A. L., \& Lazio, T. J. W. 1998, ApJ, 496, 253

Cognard, I., Bourgois, G., Lestrade, J.-F., Biraud, F., Aubry, D., Darchy, B., \& Drouhin, J.-P. 1993, Nature, 366, 320

Cordes, J. M. 1990, in Low Frequency Astrophysics from Space, ed. N. E. Kassim \& K. W. Weiler (Berlin: Springer), 165

Cordes, J. M., \& Lazio, T. J. 1991, ApJ, 376, 123

Cordes, J. M., Pidwerbetsky, A., \& Lovelace, R. V. E. 1986, ApJ, 310, 737

Cordes, J. M., \& Rickett, B. J. 1998, ApJ, 507, 846

Dickey, J., \& Lockman, F. J. 1990, ARA\&A, 28, 215

Fey, A. L., \& Charlot, P. 1997, ApJS, 111, 95
Fey, A. L., Clegg, A. W., \& Fiedler, R. L. 1996a, ApJ, 468, 543

Fey, A. L., Clegg, A. W., \& Fomalont, E. B. 1996b, ApJS, 105, 299

Fiedler, R. L., et al. 1987a, ApJS, 65, 319

Fiedler, R. L., Dennison, B., Johnston, K. J., \& Hewish, A. 1987b, Nature, 326, 675

Fiedler, R., Dennison, B., Johnston, K. J., Waltman, E. B., \& Simon, R. S. 1994a, ApJ, 430, 581

Fiedler, R., Pauls, T., Johnston, K. J., \& Dennison, B. 1994b, ApJ, 430, 595

Hjellming, R. M., \& Narayan, R. 1986, ApJ, 310, 768

Kellermann, K. I., \& Owen, F. N. 1988, in Galactic and Extragalactic Radio Astronomy, ed. G. L. Verschuur \& K. I. Kellermann (Berlin: Springer), 563 
Lestrade, J.-F., Rickett, B. J., \& Cognard, I. 1998, A\&A, 334, 1068

Maitia, V., Lestrade, J.-F., \& Cognard, I. 1998, ApJ, submitted

Rickett, B. J. 1990, ARA\&A, 28, 561

Romani, R. W., Blandford, R. D., \& Cordes, J. M. 1987, Nature, 328, 324

Shen, Z.-Q., et al. 1997, AJ, 114, 1999

Spangler, S. R., \& Cordes, J. M. 1988, ApJ, 332, 346

Taylor, J. H., \& Cordes, J. M. 1993, ApJ, 411, 674

Vijayanarasimha, V., Ananthakrishnan, S., \& Swarup, G, 1985, MNRAS, 212,601
Walker, M., \& Wardle, M. 1998, ApJ, 498, L125

Waltman, E. B., Fiedler, R. L., Johnston, K. J., Spencer, J. H., Florkowski, D. R., Josties, F. J., McCarthy, D. D., Matsakis, D. N. 1991, ApJS, 77, 379

Wehrle, A. E., Cohen, M. H., Unwin, S. C., Aller, H. D., Aller, M. F., \& Nicolson, G. 1992, ApJ, 391, 589 